overcome the disease it is necessary to give up a good deal of what are ordinarily reckoned the pleasures of life and to implicitly follow out rules of conduct which call for selfdenial and self-control. No one can be expected to do this unless he is satisfied that it is necessary and unless he is possessed of at least ordinary intelligence. One often hears the expression, "the curable stage of phthisis," and I wish to say that I agree with Walshe when he stated that there was no stage of the disease which is surely curable. It is misleading to preach any such doctrine as that consumption is certainly curable if the case is taken in hand early enough. The earlier a case is taken in hand the better but we can never guarantee that a cure will be effected. The most hopeful cases for any form of treatment. are those where the disease is chronic, quiescent, and of small extent. Natural tendency to fibrosis and inherent resistance to the disease are the most important elements which make for recovery. Where the disposition to arrest and repair is strong even the most injudicious treatment may appear of signal efficacy.

In conclusion, let me say that I feel that I have given a very inadequate sketch of the means available for the treatment of consumption. The subject is a very large one and it has been possible only to touch on the most important points. For the hygienic treatment I do not claim that it is the best attainable, only that it is the best so far attained. There is no finality in medicine and the treatment of to-day may be, nay, will be, vastly improved on in the coming to-morrow. However that may be no one, I am sure, will dream of attempting to cure tuberculosis on the old plan of coddling and cosseting patients in the stuffy and vitiated atmosphere which even now is associated in the minds of many as a necessary characteristic of the sickroom.

\section{THE TREATMENT OF STREPTOCOCCIC PUERPERAL FEVER BY ANTITOXIC SERUM.}

BY ALEXANDER G. R. FOULERTON, F.R.C.S. ENG., D.P.H. CANTAB.

LECTURER ON BACTERIOLOGY AND ON PUBLIC HEALTH AT THE MIDDLESEX HOSPITAL MEDICAL SCHOOL; MEDICAL OFFICER TO THE COUNTY COUNCIL OF EAST SUSSEX.

THE uncertainty of the action of antistreptococcic serum in the treatment of streptococcic infections is well recognised in practice and because of it the method of serum treatment has been discredited to a considerable extent in this class of bacterial infections. But the reasons for this uncertainty are easily explained and the complete failure of serum treatment in a large proportion of the cases of streptococcic infection in which it has been used has been inevitable under the existing conditions.

It will be remembered that the first antistreptococcic serum-that of Marmorek-was prepared from a single strain of streptococcus, and it was believed by Marmorek, Behring, and others that the serum thus prepared was specifically antitoxic in all cases of streptococcic infection. And this belief in the unity of streptococcus pyogenes and consequently in the unity of streptococcic toxins and the correlated antitoxins was so firmly held at first that a brand of antistreptococcic serum which was at one time extensively used in this country was prepared from a single strain of streptococcus which was not even derived from a human source but had been isolated from one of the lower animals.

On the other hand, many efforts have been made to differentiate separate species of streptococci and thus streptococcus erysipelatis, streptococcus longus, streptococcus brevis, streptococcus conglomeratus, the enterococcus of Thiercelin, and others, have been described; and there can be but little doubt but that between various streptococci as we isolate them from cases of disease there are often differences - as to their several morphological and cultural characteristics, as to their pathogenic action, and as to their behaviour in relation with different antistreptococcic serums when tested in vitro or by animal experiment-so distinct as to entitle us to assume that we are dealing with definite species of streptococci rather than with mere varieties of a single species, streptococcus pyogenes. And, in short, I : u ppose that at present it would be acknorledged by most bacteriologists who come into actual contact with clinical work that the term streptococcus pyogenes, as we use it, does not denominate a single species but rather is to be understood as a sort of general name which includes several species of cocci, with related varieties, which present a common similarity in their tendency to assume a streptococcal type of grouping. It is, therefore, not a matter for surprise that, applied under such conditions of uncertainty, serum treatment-in itself the most accurately specific method in the whole range of therapeutics and demanding as a necessity equal accuracy in diagnosis-should have frequently proved a failure in cases of streptococcic infection.

The unequal results which had been obtained in clinical practice by the indiscriminate use in every case of streptococcic infection of an antistreptococcic serum prepared from a single strain were soon explained by experiment in the laboratory, and at the same time the way in which the difficulty might be met was pointed out. Amongst much experimental work which has been carried out in the investigation of the question as to the identical specificity or otherwise of different strains of streptococci that of Van de Velde has been perhaps the most suggestive. Van de Velde made a careful study of 21 strains of streptococci derived from the following cases of disease: four from cases of puerperal infection, four from cases of postoperative peritonitis, four from cases of sore-throat, three from abscesses, three from cases of erysipelas, and single strains from cases of arthritis, bronchitis, and cystitis. After a full consideration of the methods of growth and other characteristics of these 21 strains Van de Velde found himself in accordance with the opinions of those who believed in the identical specificity of all varieties of streptococci. But whilst Van de Velde himself came to this conclusion I think that the results of his carefully detailed work are at least open to the opposite interpretation, and for my own part I would claim that his experiments proved such differences between various strains which he worked with as to support what has been already said as to specific differences between some of the strains of streptococci which are found as causers of disease. For the present purpose, however, and in view of the results of Van de Velde's experiments as to the influence of different antistreptococcic serums on different strains this question as to whether the streptococci which he isolated represented one or more species is of merely academic interest and turns mainly upon the precise meaning to be attached to the word "species." Van de Velde carried out a series of experiments in order to test the action of the serum of a horse which has been immunised against one particular strain of streptococcus when used against an experimental infection by another strain. He found that serum of a horse immunised against streptococcus " $\mathrm{A}$," isolated from an abscess, protected to only a slight extent against streptococcus " $P$," isolated from a case of puerperal infection; also it was found that the serum from a horse immunised against streptococcus " $\mathrm{P}$ " did not afford any protection against streptococcus "A," each serum being, of course, actively protective against the particular strain from which it was prepared. A third horse was then immunised against both strains and the serum thus prepared was found to be actively protective against both streptococcus "A" and "P." A fourth antistreptococcic serum (Marmorek's) did not afford any protection against streptococcus " $\mathrm{A}$," and other similar results were obtained with various strains of streptococci. The logical deductions from these results were obvious and Professor Denys of Louvain, at whose suggestion Van de Velde had undertaken his investigation, proceeded to prepare a polyvalent antistreptococcic serum for clinical use, the horse being immunised against strains of streptococci obtained from the most widely diverse forms of disease. And at the present time there are several brands of polyvalent antistreptococcic serum available for general use.

During the years 1896-97-98, when antistreptococcic serum was coming into common use, I gave special attention to the results obtained in the treatment of puerperal fevers, collecting a long series of cases in which one or other brand of antistreptococcic serum had been used. And leaving on one side the cases in which no bacteriological diagnosis had been made, it was obvious that the uncertainty of result which characterised the serum treatment of streptococcic infections generally prevailed also to a very marked extent in the special clinical group of cases of streptococcic puerperal fever. And whilst it was in only a minority of these cases of puerperal infection that serum treatment appeared to have 
been of real use, its beneficial action was so distinctly marked in this minority that there could be no doubt as to the specific nature of the remedy which had been employed in them. So decided and abrupt was the improvement which sometimes followed immediately on its use that it was impossible to regard the injection of the serum and the consequent lowering of temperature and general relief from symptoms otherwise than in the relationship of cause and effect. And in view of these positive results in a minority of the cases it seemed probable in the highest degree that the frequent failure in other cases which, of equal or less severity, were clinically similar must be due to the fact that the particular serum used had no specific antitoxic action with regard to the special variety of streptococcus causing the infection, or, in other words, that the serum had been prepared from a streptococcus of different pathogenic nature.

Because of various circumstances further work in connexion with this question was postponed until the early part of 1901, when, with the valuable coöperation of Dr. Victor Bonney, a systematic investigation into the bacteriology of puerperal infections was commenced. And during the last three and a half years we have examined the contents of the uterus in a considerable series of cases of fever following on either miscarriage or labour at full term. Our results will be published in detail on an early date and I will now refer to them only so far as they effect the matter immediately under consideration, and that very briefly.

In the first place, it may be said that our results have confirmed fully what has been repeatedly demonstrated since Pasteur and Doléris first called attention to the predominating influence of streptococci in the causation of puerperal fevers. The proportion of cases of puerperal fever which are the result of a streptococcic infection has been variously stated by different authorities; thus Krönig found streptococci present in 41.9 per cent. of his series of 179 cases, whilst Whitridge Williams found them in only $29 \cdot 3$ per cent. of a series of 150 . In our own work we have endeavoured to comply as far as we could with the conditions necessary for insuring scientific accuracy and we have found in a series of cases sufficiently extensive to eliminate any gross fallacy arising from accidental coincidences that the relative importance of streptococci in puerperal infections is even more marked than a consideration of previous bacteriological researches on the subject would have led us to anticipate. In brief, our own investigations up to the present time have given the following results. We have found streptococci of one sort or another in the uterus in $46 \cdot 3$ per cent. of a series of 54 cases which we have examined, and in these cases the organisms have been found under conditions which indicated that they were the primary cause of the fever. If, however, we exclude the less severe cases of fever occurring after childbirth and consider only those which either ended in death or were clinically of a distinctly serious nature we have found streptococci present as the presumable cause of disease in $62 \cdot 5$ per cent. These statements are given in general terms and will be amplified in a future joint paper with Dr. Bonney. Our investigations having been commenced with the view of improving the serum treatment of streptococcic puerperal fever, if any practicable improvement were suggested by our results, we have studied in detail various strains of streptococci which have been isolated from our cases. Up to the present time we have dealt with 25 strains of streptococci isolated from the same number of cases of puerperal fever and we have satisfied ourselves that amongst these 25 strains there is more than one distinct species as we understand that term.

After nearly two years' work at the subject we decided that we had sufficient reason for testing the use of a serum to be prepared from a horse which had been immunised against a variety of the strains which we had isolated, certain cultures were handed over to the serum laboratories of a well-known firm, and from these an antistreptococcic serum has been prepared in the ordinary way, the horse having been vaccinated with five of the strains. As yet this serum has been used in only a few cases but amongst them there have been two cases of recovery after extremely severe forms of streptococcic infection-in one case the consequence of a miscarriage, in the other following on labour at fuii term. And whilst the special value of a serum thus prepared for use in puerperal cases as against the ordinary polyvalent antistreptococcic serum hitherto used has yet to be proved, I think that a full consideration of all the circumstances justifies the opinion that if the serum treatment of streptococcic puerperal fever is to be placed on a satisfactory basis it must be by means of some such method as that which we have endeavoured to carry out.

Apart from the failure of serum treatment in puerperal fever arising from uncertainty as to the particular strain of streptococcus which is present, the question of the dosage of the serum which is employed appears to be of considerable importance. In the absence of any approximately accurate method of standardising the potency of different antistreptococcic serums it is obvious that any system of dosage must be entirely empirical ; but a review of the cases of streptococcic puerperal fever in which a serum has been used with apparent success brings out very strongly the fact that when recovery occurs in severe cases it is after large doses of serum, frequently repeated.

In clinical practice the action of antistreptococcic serum contrasts strongly with that of antidiphtheritic serum in this respect. In diphtheria the administration of a sufficient dose of antitoxic units is usually followed by immediate improvement; the improvement is sustained and generally there is no need to repeat the dose. In cases of streptococcic puerperal fever, on the other hand, the rule has been that when improvement has followed on the first injection it has been necessary to repeat the dose of serum time after time. Thus, whilst in some cases the improvement following the first injection has been as marked as anything that is seen in the antitoxic treatment of diphtheria-sometimes, perhaps, the effect has been even more striking in the case of a patient apparently moribund with a severe streptococcic infection-it has been found again and again that within 24 hours serious symptoms have recurred, again to disappear on the repetition of the dose of serum. Regarding the matter entirely from the clinical point of view it would seem that the action of an antistreptococcic serum is purely antitoxic and that, at any rate under the conditions commonly prevailing in a severe puerperal infection, the streptococci themselves are not affected by its use. In such a case there will frequently be a condition of streptococcic pelvic cellulitis and the infecting bacteria unaffected by the serum given continue to elaborate their specific toxins. And in order to neutralise the toxins which are thus being continuously thrown into the patient's circulation repeated doses of the serum must be given until the infection comes to a natural termination.

With any of the antistreptococcic serums at present in use it is advisable to commence treatment with an injection of at least 20 cubic centimetres and one must be prepared to repeat this dose, if necessary, at least every 24 hours. Another point which appears clearly when one considers the general results of serum treatment in these cases is that if the serum is going to do any good at all the effects will be at once apparent. And consequently it is useless to persist in the use of a particular antistreptococcic serum unless its beneficial action is almost immediately apparent. If in a case of streptococcic puerperal infection no improvement follows two doses of 20 cubic centimetres administered within 12 hours it is useless to persist in administering it ; another brand of serum should at once be tried.

In conclusion I may just refer to the prejudice against the use of large injections of antistreptococcic serum which has arisen in the minds of some practitioners from the idea that the use of this class of serum is especially liable to be followed by certain toxic symptoms, the appearance of cutaneous eruptions with slight rise of temperature, and transient arthropathies. Such unfavourable symptoms are common to the use of any kind of horse serum and, other things being equal, are not more likely to follow on the injection of an antistreptococcic serum than on the injection of an antidiphtheritic serum or on the injection of a normal horse serum; in fact, the most severe constitutional disturbance which I have ever seen following the use of a serum was in a patient who had received an injection of what was for practical purposes a normal horse serum. The reason why these symptoms have been more especially noticed in the serum treatment of streptococcic infections is doubtless because in such cases it is, as I have said, frequently necessary to give large and repeated doses; and possibly also the patients who require treatment with antistreptococcic serum are in such a condition that they are specially liable to react to horse serum. In any case it is probable that these toxic symptoms following on the use of a serum are not of any particular consequence and I am not aware of a single case in which any definite harm to the patient 
has resulted. But whatever the dangers are they must be risked when dealing with a severe streptococcic infection and it cannot be too strongly insisted on that with the serums which are at present available a large dose is necessary for any success; the dose of five cubic centimetres of antistreptococcic serum which is sometimes advised is probably quite useless and failure which is the consequence of inadequate dosage is one of the reasons why the use of antistreptococcic serum in puerperal cases has fallen into partial disrepute.

The Middlesex Hospital, W.

\section{A CASE OF STREPTOCOCCIC PUERPERAL INFECTION TREATED WITH A SPECIAL PUERPERAL ANTISTREPTOCOCCIC SERUM ; RECOVERY.}

BY THOMAS ROSE, M.R.C.S., L.R.C.P.,

RESIDENT MEDICAL OFFICER AT THE CHELSEA HOSPITAL FOR WOMEN.

THE case to which the following notes refer was one of an extremely severe type of streptococcic infection in which there seemed to be no doubt that ultimately recovery was due to the action of an antistreptococcic serum.

The patient, who was 29 years of age, was admitted into the Chelsea Hospital for Women on Nov. 12th, 1904. Her previous history showed that on Oct. 15th she was delivered of her third child. The puerperium and labour were normal but the latter was a little prolonged. She was attended by a midwife, and on Oct. 18 th the temperature rose to $102 \cdot 4^{\circ} \mathrm{F}$. and the pulse-rate was 120 . A medical practitioner was then called in and the uterus was explored but nothing was found retained. An intra-uterine douche of perchloride of mercury ( 1 to 4000) was given. The temperature fell and everything appeared to be going on well until Nov. 1st, when the left leg became painful and began to swell. The temperature gradually rose again and on the 5th the right leg also was swollen. When admitted into hospital on the 12 th the patient was extremely ill; she was very anæmic, her temperature was $102^{\circ}$, and the pulse-rate was 120 . There was some rigidity of the lower part of the abdominal wall but no distension or tenderness on pressure. By vaginal examination it was found that the uterus was slightly enlarged and tender; the os uteri had contracted; there was no discharge or offensive odour. There was distinct fulness in both lateral fornices. Both legs were considerably swollen and presented typically the appearance of white leg. On the 17 th the temperature fell to $98 \cdot 4^{\circ}$, the pulse-rate was 120 , but the general condition of the patient was not improved in any way. Towards the evening the temperature began to rise again and a condition of mental apathy became apparent. She gradually became worse and on the 20th the temperature was $102 \cdot 8^{\circ}$ and the pulse-rate was 160 . On the 22 nd the patient, having been delirious for two days, passed into an unconscious condition. There were sordes on the lips and urine and fæces escaped incontinently; the temperature was $102^{\circ}$ and the pulse was uncountable. She was being fed through a nasal tube and cardiac stimulants were given freely, without, however, any improvement in the state of the pulse.

Dr. W. F. V. Bonney saw the patient on this date and advised the injection of the puerperal antistreptococcic serum suggested by Mr. A. G. R. Foulerton and doses of five cubic centimetres each were injected at noon, at 6 P.M., and at midnight. On the morning of the 23rd the temperature had fallen to $101^{\circ}$ and the pulse-rate was 148 . At noon a dose of 20 cubic centimetres of the serum was injected and at 2 A.M. on the 24th the temperature had fallen 'to $98.2^{\circ}$ and the pulse-rate was 108 . There was a most marked improvement in her general condition and she was able to take a little nourishment by the mouth. The temperature remained at the normal and progress towards recovery was uninterrupted except by the appearance of an erythematous rash on Dec. 2nd accompanied by some febrile disturbance. The rash appeared first on the abdomen, round the place at which the serum had been injected, and spread to the legs and arms; it persisted for three days, during which the temperature ranged between $98 \cdot 2^{\circ}$ and $99 \cdot 8^{\circ}$.

In this case the effect of tive serum injections was well marked. On Nov. 22nd, when the serum was first given, the patient was in a most serious condition and a fatal result was anticipated. Owing to a misapprehension rather small doses of the serum were given during the first 12 hours but distinct improvement was obvious, the temperature fell somewhat, and the pulse, hitherto uncountable, could be counted. The result of the larger dose, given 24 hours after the first injection, was very distinct, and within a few hours the patient was practically out of danger.

The case appeared to be one of pure streptococcic infection. At the commencement of the serum treatment a sterilised "swab" was passed with the usual precautions directly into the cavity of the uterus and the result of the bacteriological examination in the laboratories of the Middlesex Hospital was to prove the presence of a streptococcus in pure culture.

My thanks are due to Dr. W. H. Fenton for his kind permission to publish the details of this case, which was under his care at the hospital, and to Mr. Foulerton for placing at my disposal the special puerperal antistreptococcic serum which was used.

Chelsea Hospital for Women, S.W.

\section{ON THE ADMINISTRATION OF ANTI- STREPTOCOCCIC SERUM.'}

By J. W. THOMSON WALKER, M.B. EDIN., F.R.C.S. ENG., ASSISTANT SURGEON TO THE NORTH-WEST LONDON HOSPITAL AYD TO ST. PETER'S HOSPITAL FOR STONE AND URINART DISEASES ; LATE BACTERIOLOGIST AND PATHOLOGIST TO THE PADDINGTON GREEN CHILDREN'S HOSPITAL.

SERUM-THERAPY in many of its phases has hardly yet emerged from the experimental stage. Although recently the number of antibodies has increased and careful experiments on animals have proved their efficiency, yet there is great need for more complete observations on the effect of these serums on infective processes in the human body. We have not yet left the stage at which isolated reports of cases treated with antitoxins help towards the definition of our knowledge and each carefully observed case which is complete from the bacteriologist's point of view is of value. The following case is a record of my personal experience as a culture medium for the streptococcus. I believe that there are some points of definite value in the course and treatment of the case and have therefore ventured to give it publicity.

The source of infection was the body of a child one month old, on which a necropsy was performed on May 4th, and in which, besides supporative epiphysitis and necrosis of the right humerus, there were infected areas in the subcutaneous cellular tissues over the left pectoral muscle, the left wristjoint, around the left tendo Achillis, and the right popliteal space. These areas had either been incised and were dry and non-granulating or they formed reddened patches on the surface which showed an accumulation of bloody serum in the cellular tissue on incision. The child had been infected by way of the umbilical vein, for there was an encapsulated accumulation of yellow pus of about the size of a hazel nut at the transverse fissure of the liver.

No suspicion was entertained at the time of the necropsy that the hands had been cut or scratched during its performance. The same night, however, a tiny puncture was discovered at the distal joint fold of the left index finger and a carbolic dressing was applied. A small swollen area was present around this puncture next morning, but there were no signs of its spreading or of its absorption into the general circulation. At mid-day, rather less than 24 hours after the infection, there was a feeling of malaise which increased and three hours later a well-marked rigor occurred; the temperature at 4.30 P.M. was $97^{\circ} \mathrm{F}$. ; the axilla was now painful and on examination the lymphatics could be traced in red streaks the whole length of the arm. The axillary glands were tender but only slightly enlarged. Half an hour later the temperature had risen to $101^{\circ}$ and at 6.30 P.M. Mr. F. F.

1 Nearly three years have elapsed since this record was written but during this time the manuscript has been unaroidably laid aside. Further cases have been recorded in the interval but I believe that there are still points of sufficient interest in this one to merit publication and I have left $m y$ original comments on the case unaltered. 